Bangladesh J. Sci. Ind. Res. 42(1), 15-28, 2007

\title{
Real Adoption Impact Measure of Tomato Technologies on Production at Farmers' Level in Bangladesh
}

\author{
S. C. Barman \\ Agril. Economics and Statistics Section, HRC, BARI, \\ Joydebpur, Gazipur 1701, Bangladesh
}

\begin{abstract}
The study of real adoption impact (RAI) measure of technologies on tomato production at farmers' level revealed that there was a positive and significant relationship between yield and the values of RAI which was calculated utilizing the degrees of deviations between standard recommendations for tomato and their level of application at farmers' field. This study implies that the higher value of RAI resulting from more proper adoption of tomato technologies would give higher yield. It was found from the estimated model that 1 percent higher value of RAI would give more than 4 percent greater tomato yield. From this model it was also found that the expected yield gap and observed yield gap of tomato were much greater than that of estimated yield and observed yield indicating a scope to increase the yield of tomato. These results exclusively indicate to a situation where tomato farmers were in complete knowledge of the technologies but they were not using recommended doses of inputs and also management practices properly and completely in production system. As a result they could not reap the yield like research station yield of $80 \mathrm{t} / \mathrm{ha}$. The farmers were getting about $1 / 4$ th yield on an average and made a sharp and big yield gap of tomato between on station and farmers' field. It calls for an intensive extension work focused on tomato technologies and timely support with required inputs with a view to minimize the yield gap for national interest.
\end{abstract}

\section{Introduction}

Different standard recommendations related to different technologies in agricultural sector are available for different crops. It is also usual and has been observed that the farmers are deviating from recommendations in adopting technologies due to their different level of technical know-how and resource endowment. In case of tomato (Lycopersion esculentum Mill) crop production and adoption of different technologies, there is no exception in Bangladesh. Tomato is a nutritious vegetable crop generally grown in the winter season (December April) in Bangladesh. It is the most popular 
vegetable and acts as a source of vitamins and minerals (Table I). It contains a number of nutritive elements almost double compared to fruit apple and shows superiority with regard to food values. It is consumed as a raw salad, cooked or as processed food item such as Sauce, Ketchup, Jam, Jelly, Pickles Soup etc. Tomato has originated in Peruvian and Maxican regions and come from tropical America and spread to other parts in the World in 16th century. It was perhaps introduced into Indian Sub- Continent by the Portuguese (Das. 1993). However, it is grown both at homestead gardens for fresh consumption and commercially at the field in many parts of Bangladesh. The products produced domestically can not meet up the year round demand of the consumers in the market and also at the processing factories especially in the summer there is a demand and showed higher market price in the country. The consumption and utilization level side by side demand for tomato have been increasing day by day. This higher demand and market price have drawn attention of the government and expert groups who have started realizing its importance. So, to make available tomato year round domestically some exotic varieties of tomato have been selected through research and development program during last decade. To meet up local demand Bangladesh Government have also been importing tomato from the neighboring countries. Government imported $9395.14 \mathrm{~m}$. $\mathrm{t}$ of tomato in exchange of TK. 1503 millions from foreign countries in the year 2000-2001
(BBS. 2004).

It was found that about 120 thousand m. $\mathrm{t}$ of tomato was utilized at national level in Bangladesh as estimated from the time series data in 2003-2004 and it was obtained only from domestic production. It was also observed from this data on tomato consumption over 12 years that the quantity utilized has been increasing with a positive growth rate of $7 \%$ per annum and the coefficient of variation was about 11 percent. The average wholesale price of tomato $(\mathrm{Tk} / \mathrm{m}$. t) during this period was also increased with a growth rate of positive 13.6 percent per annum and its variation was in high order with a CV. of about 56 \% year to year (Barman and Shiblee, 2001-2005). It was also found that the demand in the Bangladesh market for tomato has been increasing and year to year the quantity imported varied by a CV. of 172 percent and also have very low yield of 6.69 t/ha at national level in 2003-2004. One of the main reasons for such poor yield of tomato could be attributed to farmers' general adoption of improved technologies at a lower level. On the other hand, the existing market demand for tomato in Bangladesh calls for a big effort in production system for boosting up the tomato production level. Side by side, the present situation also demands the dissemination of improved technologies of this crop to the farmers through different agencies in Bangladesh. 
Several Extension Education researches with adoption of recommended production practices by the farmers mainly in cereals and fiber crops showed that there is a positive relationship between farmers' technical know-how and adoption of agricultural innovations (Razzaque, 1977, Hossain,1981). However, real adoption impact measures of tomato technologies on production at farmers' level are lacking. As such, study has become necessary to identify the real adoption impact and the degree of deviations in adopting the technologies of tomato crops with proper weights. In this field, it is considered that mere use of any technology will not be appropriate for considering adoption of the technology and it will be better to define the adoption of a new technology as the complete and proper use of the recommended technologies or practices. Keeping this in view, a study was undertaken to measure the "Real Adoption Impact (RAI)" of tomato technologies on its production at farmers' level with the following objectives.

\section{Objectives}

i. To identify the recommended improved technologies for tomato and level of uses in cultivation at farms level.

ii. To identify the yield differences between research station yield and farmers' yield

iii. To determine the extent of adoption of technologies and measures impact on yield at farmers' level with two assumptions

a. Equal deviation would affect yield for each of the technologies when other factors of production remain constant

b. The deviation could not exceed the recommended inputs (doses) and practices for all- practical purposes.

\section{Materials and Method}

\section{Derivation of RAI}

To derive the measure of RAI an attempt was made using the deviation proportions like mean of the deviation proportion, standard deviation (s. d) of deviation proportion etc., (Spiegel M. R., 1961). However, standard deviation was seemed to be inappropriate in this respect, because cases might arise where deviation proportions were very high in magnitude with a very small scattered ness or deviation. Under this circumstance, the standard deviation value of deviation proportions was very small which implied a high impact of adoption though the actual situation was different, to specific just opposite. Therefore, it was preferred to use the mean of deviation proportions in this measure. The RAI measure derived was as followed.

Technologies are $T_{1}, T_{2},------------, ~ T_{n}$.

Inputs recommended (in proper unit) $r_{1}, r_{2},------, r_{n}$. 
Inputs used in actual practice (in proper unit) $\mathrm{P}_{1}, \mathrm{P}_{2}, \ldots \ldots ., \mathrm{P}_{\mathrm{n}}$

Absolute deviations $d_{1}=\left|r_{1}-P_{1}\right|, d_{2}=\left|r_{2}-P_{2}\right|$, ............ $\mathrm{d}_{\mathrm{n}}=\left|\mathrm{r}_{\mathrm{n}}-\mathrm{P}_{\mathrm{n}}\right|$.

Deviation proportions: $\mathrm{R}_{1}=\frac{\mathrm{d}_{1}}{\mathrm{~d}_{1}}, \mathrm{R}_{2}=\frac{\mathrm{d}_{2}}{\mathrm{r}_{2}}$, ..........., $\mathrm{R}_{\mathrm{n}}=\frac{\mathrm{d}_{\mathrm{n}}}{\mathrm{r}_{\mathrm{n}}}$,

Now Real Adoption Impact (RAI) $=\frac{\mathrm{n}}{\mathrm{N}^{-} \cdot-\frac{1}{\mathrm{R}} \overline{\mathrm{i}}}$

$$
\begin{aligned}
& =\frac{\mathrm{n}}{\mathrm{N}} \cdot \frac{1}{-1}=\frac{\mathrm{n}}{\mathrm{N}}
\end{aligned}
$$

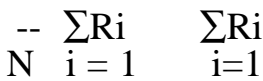

Where, $\mathrm{n}=$ number of technologies considered by the tomato farmers in practice ; $\mathrm{N}=$ number of major technologies considered in the study; and

$\mathrm{Ri}=\left(\frac{\mathrm{ri}-\mathrm{Pi}}{\mathrm{ri}}\right), \quad(\mathrm{i}=1, \quad 2, \ldots \ldots \ldots \ldots, \mathrm{N})$

Now, those farmers who are using the tomato technologies as per recommendations i.e., who are adopting technologies completely and properly, in this context, will have deviation proportions equal to zero i.e., where $\mathrm{r} i$ $=\mathrm{Pi}$ for all $(\mathrm{i}=1,2,-----\mathrm{N})$. Then all $\mathrm{Ri}=$ 0 (Zero) and leading RAI $=\alpha$ (infinity), the complete adoption case. But on the other way RAI will be 0 (zero) when $n$ is zero i.e., no adoption case. Other values of RAI will be remained within 0 (zero) to $\alpha$ (infinity). Thus the range of RAI is zero to infinity. Generally a large value of RAI will lead to a situation of more adoption of technology and RAI defined in equation (1) was used as a measure of adoption.
RAI is better in the sense that it helps to measure the degree of deviation in the farmer's actual practices from the research station recommendations. It is basically relied on the application of conventional mathematical relation in existing farmers' circumstances (Barman and Islam, 2005). After getting RAI a statistical test for the correlation between $\mathrm{RAI}(\mathrm{X})$ and observed yield (Y) of particular crop may be performed. On acceptance of a positive correlation between RAI and observed yield a simple regression equation can be fitted considering yield (Y) as a dependent and RAI (X) as the independent variable for identifying the extent of effect of RAI on tomato yield.

\section{Data collection}

To study the nature of RAI on Ratan tomato production at the farmers' level necessary data were collected from tomato farmers who cultivated Ratan tomato in four Upazillas namely Hathazari, Comilla Sodar, Bagharpara and Nagarpur in Chittagong, Comilla, Jessore and Tangail districts respectively in Bangladesh. Ratan variety of tomato is resistant to bacterial wilt and widely suited to grow in winter session in Bangladesh. It is relatively flashy compared to other tomato variety and has attractive reddish color (Anon, 2004). However, from each of the four study areas 15 tomato farmers were chosen for this study irrespective of farm size groups and taken for collective analysis for identifying average performance. So the total sample size for this study in four Upazillas consisted of 60 tomato cultivators in four dis- 
tricts who cultivated the Ratan tomato variety. Study sample was drawn in purposive simple random sampling procedure from a list of 537 tomato farmers in four districts. Data on different aspects of tomato production and level of inputs used different management practices and technology used etc, were collected through direct interview and survey method a / using a pre-tested questionnaire during the period of two and half months harvesting and after harvesting time in 2005.

Some relevant Data were also collected from secondary sources. In this study, the technologies recommended by Olericulture Division, HRC, BARI Joydebpur were listed and the average yield of Ratan tomato (80.00 t/ ha.) in this center was recorded. For this study a total of twelve recommended technologies or management practices such as number of ploughing $\left(T_{1}\right)$. number of laddering $\left(T_{2}\right)$, per hectare rate of manure $\left(T_{3}\right)$, per hectare rate of Urea $\left(\mathrm{T}_{4}\right)$, TSP $\left(\mathrm{T}_{\mathrm{s}}\right)$, MP $\left(\mathrm{T}_{6}\right)$, number of seedling per hectare $\left(T_{7}\right)$, age of seedling $\left(\mathrm{T}_{8}\right)$, number of weeding $\left(\mathrm{T}_{9}\right)$ number of irrigation $\left(\mathrm{T}_{10}\right)$, number of plant protection measures $\left(T_{11}\right)$, and per hectare dose of boron $\left(\mathrm{T}_{12}\right)$ were considered in this study. However, the technology gap between recommended and application of various technological inputs and management practices mentioned above in the study were calculated and presented in the Table II. The RAI was measured using equation (1) and a positive correction between RAI and observed yield of different tomato farms was established using statistical technique. Then a regression equation between RAI (X) and yield (Y) was obtained using following equation

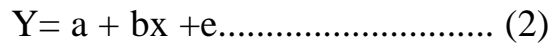

On the basis of this regression equation the expected yields $\left(\mathrm{Y}_{\text {expt }}\right)$ were obtained, then the expected yield $\left(\mathrm{Y}_{\text {expt }}\right)$ gaps and observed yield $\left(\mathrm{Y}_{\mathrm{obs}}\right)$ gaps were calculated for studied farms for useful comparisons. The regression equation (2) would be helpful to predict the yield of Ratan tomato at the farmers' field once the capacity of the farmers to follow the recommended practices for cultivation of the crop is known and consequently the suggestions would be more effective for improving the tomato yield. Some statistical tools were also used in testing the findings in fulfilling the objectives of the study.

\section{Results and Discussion}

In the study of real adoption impact measure of tomato technologies on production it was found that the farmers used about a number of eleven technologies according to their level of endeavors and financial capacity in Ratan tomato cultivation in study areas. But tomato farmers were deviated from the recommendations in several degrees in the use of management practices and technological inputs in practical operations. In some cases, especially in the use of pesticide and irrigation water, about $33 \%$ farmers

a / = Survey Upazillas and Blocks were selected with the concern of Extension Officers and Field workers in respective Upazilla. 
Table I. The nutritive value of tomato and apple (per $100 \mathrm{gm}$. of edible portion)

\begin{tabular}{|c|c|c|c|}
\hline \multirow{3}{*}{$\begin{array}{l}\text { Items and nutritive elements } \\
\text { Water }\end{array}$} & \multicolumn{3}{|c|}{ Qty } \\
\hline & \multicolumn{2}{|c|}{ Tomato $^{a}$} & \multirow{2}{*}{$\begin{array}{r}\text { Apple } \\
85.9\end{array}$} \\
\hline & 94.1 & $\mathrm{~g}$ & \\
\hline Protein & 1.0 & $"$ & 0.3 \\
\hline Fat & 0.3 & $"$ & 0.1 \\
\hline Carbohydrates & 4.0 & $"$ & 13.4 \\
\hline Fiber & 0.6 & $"$ & - \\
\hline Vitamin A & 1100.0 & $\mathrm{IU}$ & trace \\
\hline Vitamin B & 0.2 & $\mathrm{mg}$ & 120 \\
\hline Vitamin C & 27.0 & $"$ & 2 \\
\hline Nicotinic acid & 0.6 & $"$ & 0.2 \\
\hline Pantothenic acid & 0.31 & $"$ & - \\
\hline Vitamin E & 0.27 & $"$ & - \\
\hline Biotin & 0.004 & $"$ & - \\
\hline Malic acid & 150.0 & $"$ & - \\
\hline Citric acid & 390.0 & $"$ & - \\
\hline Oxalic acid & 7.5 & $"$ & - \\
\hline Sodium (Na) & 3.0 & $"$ & - \\
\hline Potassium (K) & 268.0 & & - \\
\hline Calcium (Ca) & 11.0 & $"$ & 0.01 \\
\hline Magnesium (Mg) & 11.0 & $"$ & - \\
\hline $\operatorname{Iron}(\mathrm{Fe})$ & 0.4 & $"$ & 1.7 \\
\hline Copper(Cu) & 0.1 & $"$ & - \\
\hline Manganese(Mn) & 0.19 & $"$ & - \\
\hline Phosphorus (P) & 27.0 & $"$ & 0.02 \\
\hline Sulphur (S) & 11.0 & $"$ & - \\
\hline Chlorine (Cl) & 51.0 & $"$ & - \\
\hline Mineral & - & & 0.3 \\
\hline Calorific value & - & & 56 \\
\hline Riboflavin & - & & 30 \\
\hline
\end{tabular}

Sources: $\mathrm{a}=$ Das. P.C., 1993.Vegatable Crops of India. Kalyani Publishers.1/1Rajinder Nagar,,Ludhiana 141008,India, 1stedition, p,p-1-219.

$\mathrm{b}=$ Singh. Ranjit., 1969. Fruits, National Book Trust, India. A. 5.Green Park, New Delhi 110016, PP. 1-221. 


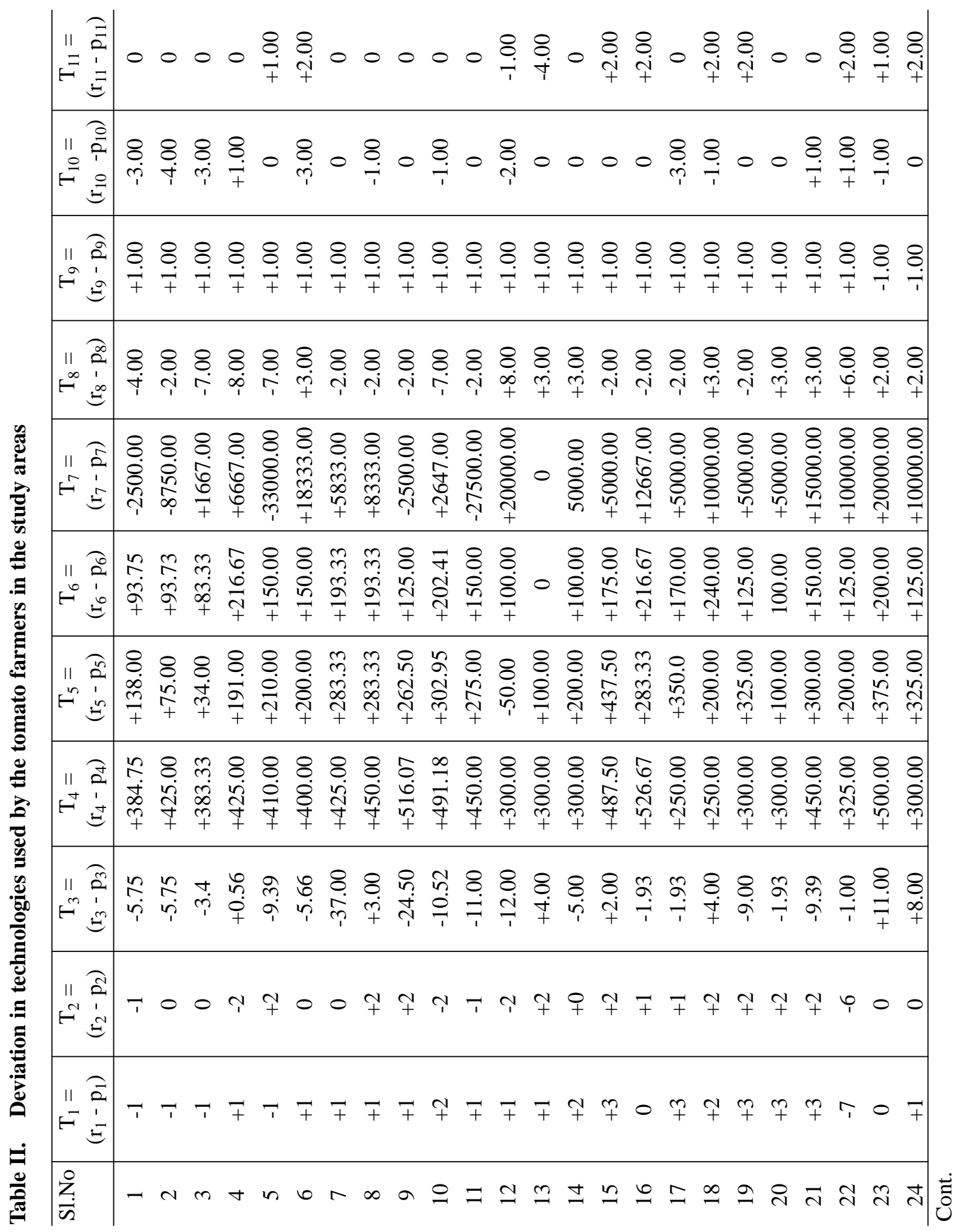




\begin{tabular}{|c|c|c|c|c|c|c|c|c|c|c|c|c|c|c|c|c|c|c|c|}
\hline 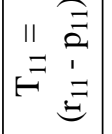 & $\begin{array}{lll}8 & 8 & 8 \\
+ & + \\
+ & +\end{array}$ & $\begin{array}{l}8 \\
+ \\
+\end{array}$ & $\begin{array}{l}8 \\
+ \\
+\end{array}$ & $\begin{array}{l}8 \\
\\
+ \\
+\end{array}$ & $\begin{array}{l}8.8 \\
+ \\
+\end{array}$ & $\stackrel{8}{8}$ & & ○ $\underset{+}{\stackrel{8}{+}}$ & $\begin{array}{l}8 \\
i \\
1\end{array}$ & & ○ $\begin{array}{l}8 \\
\dot{+} \\
+\end{array}$ & 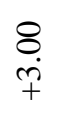 & & 0 & $b_{i}$ & & & & $\begin{array}{ll}5 & 8 \\
\dot{5} & \dot{1} \\
1 & +\end{array}$ \\
\hline 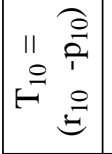 & $\underset{i}{\stackrel{8}{i} 0} \underset{i}{\stackrel{8}{i}}$ & 0 & 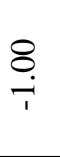 & $\begin{array}{l}8 \\
\stackrel{-}{-} \\
i\end{array}$ & $\underset{1}{8}$ & $\begin{array}{ll}8 & 8 \\
& 7 \\
1 & 7\end{array}$ & & $\begin{array}{ll}8 & 8 \\
& \stackrel{0}{1} \\
& 1\end{array}$ & 0 & - & 00 & $\stackrel{8}{\stackrel{8}{+}}$ & $\begin{array}{l}8 \\
\dot{0}\end{array}$ & $\begin{array}{l}8 \\
\stackrel{+}{+} \\
+\end{array}$ & b & & & & S. \\
\hline $\begin{array}{ll}\| & \overparen{2} \\
\sigma & 1 \\
\sigma & 0 \\
0\end{array}$ & $\begin{array}{lll}8 & 8 & 8 \\
+ & + & \dot{1} \\
+ & 1\end{array}$ & \& & $\begin{array}{l}8 \\
+ \\
+\end{array}$ & $\begin{array}{l}8 \\
+ \\
+\end{array}$ & $\circ c$ & & & $\begin{array}{ll}8 \\
8 \\
\rightarrow & + \\
+\end{array}$ & $\begin{array}{l}8 \\
+ \\
+\end{array}$ & $\begin{array}{l}8 \\
\stackrel{+}{+}\end{array}$ & $\begin{array}{l}8 \\
\stackrel{8}{0} \\
+ \\
+ \\
+\end{array}$ & $\underset{+}{\stackrel{8}{+}}$ & 8. & $\circ$ & $p_{i}$ & & & & $\begin{array}{cc}B & 8 \\
i & + \\
i & +\end{array}$ \\
\hline 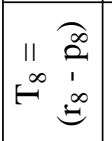 & $\begin{array}{lll}8 & 8 & 8 \\
\infty & \dot{1} & 8 \\
\infty & \dot{+} \\
+ & + & +\end{array}$ & $\begin{array}{l}8 \\
\dot{1}: \\
+\end{array}$ & $\begin{array}{l}8 \\
\stackrel{8}{+}\end{array}$ & $\stackrel{8}{\stackrel{\leftrightarrow}{+}}$ & $\begin{array}{ll}8 & 8 \\
\stackrel{0}{0} & 5\end{array}$ & $\begin{array}{ll}8 & 8 \\
+ & 9 \\
+ & -\end{array}$ & $\begin{array}{l}8 \\
\stackrel{8}{+} \\
+\end{array}$ & $\begin{array}{ll}8 & 8 \\
0 & 8 \\
& +\end{array}$ & $\begin{array}{l}8 \\
\dot{0} \\
+\end{array}$ & $\begin{array}{l}\stackrel{8}{+} \\
+\end{array}$ & $\begin{array}{ll}\stackrel{8}{8} & 8 \\
\dot{m} & \dot{1}\end{array}$ & $\begin{array}{l}8 \\
\text { i }\end{array}$ & $\begin{array}{l}8 \\
\stackrel{8}{1} \\
\stackrel{1}{1}\end{array}$ & 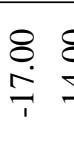 & 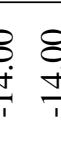 & & & & $\begin{array}{ll}0 & 8 \\
& 8 \\
1 & 1\end{array}$ \\
\hline 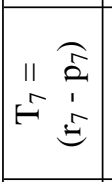 & 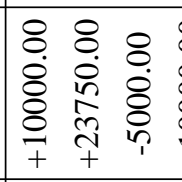 & $\begin{array}{l}8 \\
8 \\
\circ \\
8 \\
8 \\
\circ \\
+ \\
+\end{array}$ & $\begin{array}{l}8 \\
\stackrel{0}{8} \\
\stackrel{8}{8} \\
\stackrel{\leftrightarrow}{+}\end{array}$ & $\begin{array}{l}8 \\
8 \\
8 \\
8 \\
8 \\
+ \\
+\end{array}$ & $\begin{array}{l}8 \\
8 \\
\dot{8} \\
8 \\
8 \\
10 \\
+\end{array}$ & $\begin{array}{ll}8 & \\
8 & \\
8 & 0 \\
8 & 0 \\
10 & \\
+ & \end{array}$ & $\begin{array}{l}8 \\
8 \\
0 \\
0 \\
0 \\
0 \\
+1 \\
+\end{array}$ & \begin{tabular}{ll}
8 & 8 \\
0 & 0 \\
0 & 8 \\
0 & 8 \\
\hdashline & 8 \\
1 & 7
\end{tabular} & $\begin{array}{l}8 \\
8 \\
8 \\
\stackrel{0}{1} \\
+ \\
+\end{array}$ & $\begin{array}{l}8 \\
\dot{8} \\
\dot{m} \\
0 \\
\infty \\
+ \\
+\end{array}$ & $\begin{array}{ll}8 & 8 \\
0 & 0 \\
\dot{8} & 8 \\
1 & 8 \\
& 8 \\
+ & + \\
+\end{array}$ & $\begin{array}{l}8 \\
8 \\
8 \\
0 \\
+ \\
+\end{array}$ & $\begin{array}{l}8 \\
8 \\
8 \\
8 \\
8 \\
+1 \\
+1\end{array}$ & $\begin{array}{l}8 \\
8 \\
\dot{8} \\
8 \\
8 \\
\cdots \\
7 \\
7\end{array}$ & 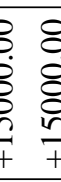 & b & 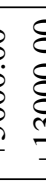 & & 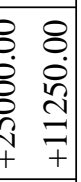 \\
\hline $\begin{array}{cc}11 & 0 \\
0 & 0 \\
0 & 0 \\
0 & 0\end{array}$ & 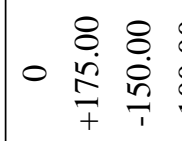 & 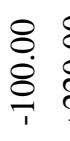 & 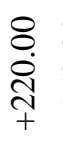 & $\begin{array}{l}8 \\
8 \\
\text { in } \\
+ \\
+\end{array}$ & $\begin{array}{l}8 \\
\stackrel{8}{0} \\
8 \\
\\
+ \\
+\end{array}$ & $\begin{array}{l}8 \\
8 \\
0 \\
\text { in } \\
+\end{array}$ & 0 & 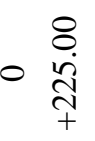 & $\begin{array}{l}8 \\
\stackrel{8}{0} \\
\stackrel{7}{+} \\
+\end{array}$ & $\begin{array}{l}8 \\
8 \\
0 \\
\text { 늠 } \\
+\end{array}$ & $\begin{array}{ll}8 & 8 \\
10 & 0 \\
& 10 \\
+ & +\end{array}$ & $\begin{array}{l}8 \\
8 \\
\infty \\
+ \\
+ \\
+\end{array}$ & $\begin{array}{l}8 \\
8 \\
0 \\
10 \\
+ \\
+\end{array}$ & $\begin{array}{l}8 \\
8 \\
\circ \dot{0} \\
\text { 1े } \\
+\end{array}$ & 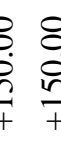 & & & & $\begin{array}{ll}0 & 8 \\
\dot{5} & 0 \\
+ & 1 \\
& 1\end{array}$ \\
\hline 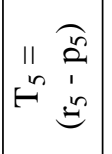 & $\begin{array}{llll}8 & & & \\
\dot{8} & 0 & 0 \\
\stackrel{1}{1} & & & \end{array}$ & $\circ$ & $\begin{array}{l}8 \\
\stackrel{0}{\dot{0}} \\
\stackrel{+}{+} \\
+\end{array}$ & $\begin{array}{l}8 \\
\stackrel{8}{0} \\
\stackrel{0}{1} \\
\stackrel{4}{+} \\
+\end{array}$ & 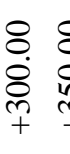 & $\begin{array}{l}8 \\
\stackrel{8}{0} \\
\stackrel{0}{m} \\
+ \\
+\end{array}$ & 0 & 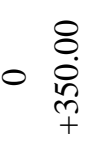 & 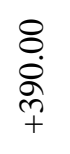 & $\begin{array}{l}8 \\
\stackrel{8}{1} \\
\stackrel{1}{+} \\
+\end{array}$ & 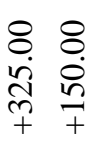 & $\begin{array}{l}8 \\
\dot{0} \\
\stackrel{+}{+}\end{array}$ & $\begin{array}{l}\stackrel{8}{\circ} \\
\stackrel{0}{\stackrel{\leftrightarrow}{1}} \\
+\end{array}$ & 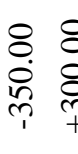 & $\begin{array}{l}5 \\
0 \\
0 \\
0 \\
0 \\
0 \\
+ \\
+\end{array}$ & & & & 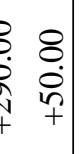 \\
\hline 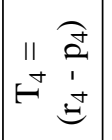 & 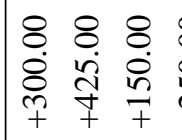 & $\begin{array}{l}8 \\
\stackrel{8}{0} \\
\stackrel{0}{1} \\
\stackrel{1}{+} \\
+\end{array}$ & $\begin{array}{l}8 \\
\dot{8} \\
\dot{0}\end{array}$ & 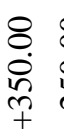 & 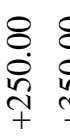 & $\begin{array}{l}8 \\
\stackrel{8}{0} \\
\stackrel{1}{10} \\
\stackrel{1}{+} \\
+\end{array}$ & 0 & 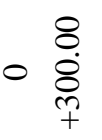 & $\begin{array}{l}8 \\
8 \\
\dot{0} \\
\stackrel{+}{+} \\
+\end{array}$ & $\begin{array}{l}8 \\
\text { L } \\
\stackrel{1}{m} \\
+\end{array}$ & 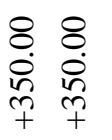 & 0 & $\begin{array}{l}8 \\
8 \\
\dot{0} \\
\stackrel{1}{0} \\
+\end{array}$ & 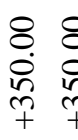 & 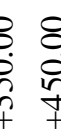 & & & & 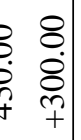 \\
\hline 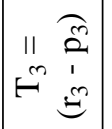 & $\begin{array}{lll}8 & & 8 \\
\dot{P} & \circ & \stackrel{1}{1}\end{array}$ & $\begin{array}{ll}\text { ㅇำ } \\
0 \\
0\end{array}$ & 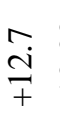 & $\begin{array}{l}8 \\
0 \\
\dot{0} \\
+\end{array}$ & $\begin{array}{l}8 \\
\stackrel{8}{1} \\
+ \\
+\end{array}$ & 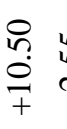 & 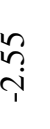 & 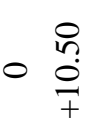 & $\begin{array}{l}R \\
\stackrel{R}{p}\end{array}$ & $\begin{array}{l}\text { 오 } \\
\text { 1ִ } \\
\text { †े } \\
+\end{array}$ & $\begin{array}{ll}\overrightarrow{1} & \\
\dot{\infty} & 0 \\
\stackrel{+}{+} & 0\end{array}$ & 0 & $\begin{array}{l}8 \\
\stackrel{8}{+\infty} \\
+\end{array}$ & & $\begin{array}{ll}0 & 1 \\
0 & \infty \\
0 & +\end{array}$ & & & & 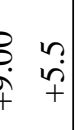 \\
\hline 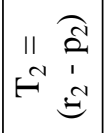 & 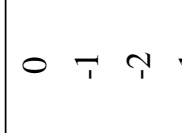 & $\rightarrow c$ & $\tau$ & 0 & $7 c$ & 0. & 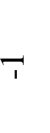 & $\neg \Upsilon$ & $P$ & $Y_{i}$ & $\underset{1}{+} \underset{+}{\sim}$ & 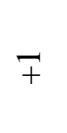 & 7 & $F$ & +7 & & & & $\begin{array}{rr}1 & N\end{array}$ \\
\hline 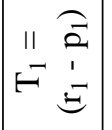 & 7 & 0 & 7 & $F$ & 0 & 7 & T & $\Upsilon$ & $\vec{r}$ & 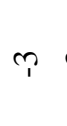 & 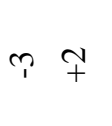 & $F$ & $\stackrel{m}{+}$ & $\stackrel{\sim}{+}$ & $n \mp$ & & & & \\
\hline ż & $\mathscr{\sim} \curvearrowright$ & $\stackrel{\sim}{\sim}$ & 2 & $C_{C}$ & & & & & & & & ㅇ & $f$ & F & y & & & & \& \\
\hline
\end{tabular}




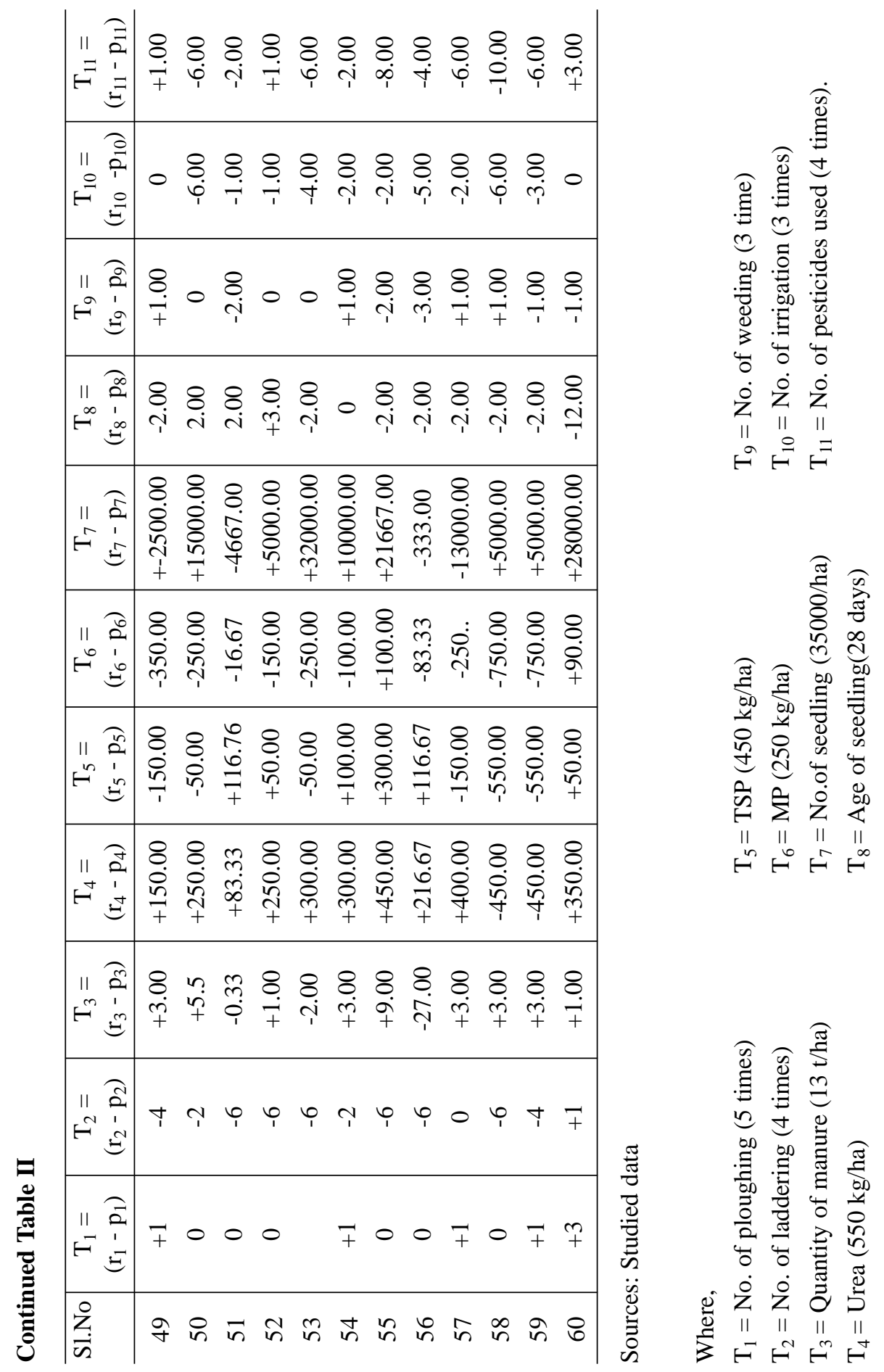


followed recommended doses having zero deviation value (Table II). Higher degree of deviation was observed with regard to the use of purchased inputs like fertilizer, seedlings and also manure etc, and the positive sign associated with individual deviation figure indicated that the tomato farmers were using less than that of the recommended level. While the negative sign associated with respective deviation figure reflected a level of input use slightly more dose in actual practices than the recommended doses. This deviation in the application of management practices and inputs use affected the tomato yield at the farmers' field.

However, a relationship was observed between yield of tomato and calculated value of RAI. In establishing the correlation between RAI (X) and observed yield (Y) of tomato, the value of $\mathrm{r}$ (correlation coefficient) was calculated and it was observed that $r=0.75$. For testing the positiveness of value of $r$, statistical test was done taking t- distribution and the null hypothesis $\mathrm{H}_{0}=0$, and tested it against the alternative hypothesis $\mathrm{H}_{1}$, $>0$ at 1 percent level of significance. In t-distribution with (n-2) degrees of freedom (d. f) under 'Ho' with n number of paired observations $(x, y)$ and $r$, the value of correlation coefficient, it was found that the $\mathrm{t}$ - observed (= 8.593) which was greater than the tabular $\mathrm{t}_{01},{ }_{58}(=2.688)$. So, the null hypothesis Ho is rejected and the alternative hypothesis $\mathrm{H}_{1}$ is accepted. i. e, there was a positive correlation between RAI and yield of the studied tomato farms. Therefore, on the acceptance of alter- native hypothesis $\mathrm{H}_{1}$, a positive correlation between RAI and observed yield of tomato on studied farms was established. Now the regression equation between the value of RAI and Ratan tomato yield was obtained as followed.

$$
\begin{aligned}
& \mathrm{Y}=8.869+4.336^{* * *}(\mathrm{X}) \\
& \mathrm{SE}=\quad(0.5046) \\
& \mathrm{t}-\text { value }=8.593, \quad \mathrm{r}=0.75, \quad \mathrm{R}^{2}=0.56
\end{aligned}
$$

The positive and significant coefficient at 1 percent level in the above estimated regression indicated that higher value of RAI (for X in this case) resulted from more accurate and proper adoption of tomato technologies and management practices in practical operation would give higher yield of tomato (column 4 in Table III). This fitted regression model also indicated that if the tomato producers could increase the value of RAI by 1 percent using recommended tomato production technology packages completely and properly in production system would get more than 4 times higher yield. It revealed that the yield of tomato is significantly influenced by real adoption of technological inputs and management practices in production.

On the basis of this estimated regression model the expected tomato yields ( $\mathrm{Y}_{\text {expts }}$ ) were obtained and than the expected yield gaps and observed yield gaps of tomato were calculated for the studied farms and made useful comparison among them (Table III). It was found that the expected yield gaps and observed yield gaps of the tomato farms were

*** Significant at $1 \%$ level. 
Table III. Regression equation for estimation of expected yield gap and observed yield gap of tomato farms in the study areas

\begin{tabular}{|c|c|c|c|c|c|}
\hline $\begin{array}{l}\text { SL No } \\
\text { (1) }\end{array}$ & $\begin{array}{l}\text { Yield (t/ha) } \\
\qquad(\mathrm{Y}) \\
(2)\end{array}$ & $\begin{array}{l}\text { RAI value } \\
\text { ( X ) } \\
\text { (3) }\end{array}$ & $\begin{array}{l}\text { Expected yield } \\
\qquad \text { (Y expt) } \\
\text { (4) }\end{array}$ & $\begin{array}{c}\text { Expected yield } \\
\text { gap (Y res -Yexpt) } \\
\text { (5) }\end{array}$ & $\begin{array}{c}\text { Observed yield } \\
\text { gap (Yres - Yobs) } \\
\text { (6) }\end{array}$ \\
\hline 1 & 27.30 & 3.90 & 25.78 & 54.22 & 52.7 \\
\hline 2 & 25.05 & 2.80 & 21.01 & 58.99 & 54.95 \\
\hline 3 & 35.15 & 5.00 & 30.55 & 49.45 & 44.85 \\
\hline 4 & 20.21 & 2.79 & 20.97 & 59.03 & 59.79 \\
\hline 5 & 29.86 & 2.20 & 18.41 & 61.59 & 50.14 \\
\hline 6 & 22.80 & 2.85 & 21.23 & 58.77 & 57.2 \\
\hline 7 & 27.68 & 1.90 & 17.11 & 62.89 & 52.32 \\
\hline 8 & 21.77 & 2.67 & 20.45 & 59.55 & 58.23 \\
\hline 9 & 21.60 & 2.17 & 18.28 & 61.72 & 58.4 \\
\hline 10 & 29.36 & 2.17 & 18.28 & 61.72 & 50.64 \\
\hline 11 & 27.10 & 2.43 & 19.41 & 60.59 & 52.9 \\
\hline 12 & 11.50 & 2.30 & 18.84 & 60.16 & 68.5 \\
\hline 13 & 35.20 & 5.61 & 33.19 & 46.81 & 44.8 \\
\hline 14 & 20.00 & 4.00 & 26.21 & 53.79 & 60.00 \\
\hline 15 & 22.16 & 2.27 & 18.71 & 61.29 & 57.84 \\
\hline 16 & 20.67 & 2.67 & 20.45 & 59.55 & 59.33 \\
\hline 17 & 25.52 & 3.19 & 22.70 & 57.3 & 54.48 \\
\hline 18 & 22.00 & 3.38 & 23.52 & 56.48 & 58.00 \\
\hline 19 & 12.25 & 2.39 & 19.23 & 60.77 & 67.75 \\
\hline 20 & 22.25 & 3.67 & 24.78 & 22.00 & 57.75 \\
\hline 21 & 17.00 & 2.16 & 18.23 & 61.77 & 63.00 \\
\hline 22 & 15.60 & 1.78 & 16.59 & 63.41 & 63.4 \\
\hline 23 & 14.08 & 2.23 & 18.54 & 61.46 & 65.92 \\
\hline 24 & 12.50 & 2.91 . & 21.49 & 58.51 & 67.5 \\
\hline 25 & 23.00 & 3.78 & 25.26 & 54.74 & 57.00 \\
\hline 26 & 25.00 & 3.48 & 23.96 & 56.04 & 55.00 \\
\hline 27 & 25.45 & 3.74 & 25.09 & 54.91 & 54.55 \\
\hline 28 & 35.25 & 5.02 & 30.64 & 49.36 & 44.75 \\
\hline 29 & 17.95 & 2.15 & 18.19 & 61.81 & 62.05 \\
\hline 30 & 17.46 & 2.48 & 19.62 & 60.38 & 62.54 \\
\hline
\end{tabular}




\section{Continued Table III.}

\begin{tabular}{|c|c|c|c|c|c|}
\hline (1) & $\begin{array}{l}\text { Yield (t/ha) } \\
\qquad(\mathrm{Y}) \\
(2) \\
\end{array}$ & $\begin{array}{l}\text { RAI value } \\
\text { ( X ) } \\
(3) \\
\end{array}$ & $\begin{array}{c}\text { Expected yield } \\
\text { (Y expt) } \\
(4)\end{array}$ & $\begin{array}{c}\text { Expected yield } \\
\text { gap (Y res -Yexpt) } \\
(5)\end{array}$ & $\begin{array}{c}\text { Observed yield } \\
\text { gap (Yres - Yobs) } \\
(6) \\
\end{array}$ \\
\hline 31 & 15.50 & 3.40 & 23.61 & 56.39 & 64.5 \\
\hline 32 & 11.50 & 2.63 & 20.27 & 59.73 & 68.5 \\
\hline 33 & 40.25 & 7.86 & 42.95 & 37.05 & 39.75 \\
\hline 34 & 38.62 & 5.95 & 34.67 & 45.33 & 41.38 \\
\hline 35 & 13.50 & 1.99 & 17.50 & 62.5 & 66.5 \\
\hline 36 & 27.52 & 2.19 & 18.36 & 61.64 & 52.48 \\
\hline 37 & 17.04 & 2.13 & 18.10 & 61.9 & 62.96 \\
\hline 38 & 23.67 & 2.81 & 21.05 & 58.95 & 56.33 \\
\hline 39 & 18.52 & 2.28 & 18.76 & 61.24 & 61.48 \\
\hline 40 & 20.40 & 2.24 & 18.58 & 61.42 & 59.6 \\
\hline 41 & 17.20 & 1.48 & 15.29 & 64.71 & 62.8 \\
\hline 42 & 21.25 & 1.97 & 17.41 & 62.59 & 58.75 \\
\hline 43 & 20.70 & 2.10 & 17.97 & 62.03 & 59.3 \\
\hline 44 & 22.80 & 2.52 & 19.80 & 60.2 & 57.2 \\
\hline 45 & 12.52 & 1.36 & 14.77 & 65.23 & 67.48 \\
\hline 46 & 10.76 & 1.22 & 14.16 & 65.84 & 69.24 \\
\hline 47 & 16.00 & 2.00 & 17.54 & 62.46 & 64.00 \\
\hline 48 & 19.85 & 2.76 & 20.84 & 59.16 & 60.15 \\
\hline 49 & 27.86 & 3.49 & 24.00 & 56.00 & 52.14 \\
\hline 50 & 20.00 & 2.01 & 17.58 & 62.42 & 60.00 \\
\hline 51 & 24.47 & 2.96 & 21.70 & 58.3 & 55.53 \\
\hline 52 & 40.42 & 3.08 & 22.22 & 57.78 & 59.58 \\
\hline 53 & 12.60 & 1.80 & 16.67 & 63.33 & 67.4 \\
\hline 54 & 22.50 & 2.83 & 21.14 & 58.86 & 57.5 \\
\hline 55 & 10.45 & 1.36 & 14.77 & 65.23 & 69.55 \\
\hline 56 & 14.18 & 1.74 & 16.41 & 63.59 & 65.82 \\
\hline 57 & 18.36 & 2.48 & 19.62 & 60.38 & 61.64 \\
\hline 58 & 9.44 & 0.93 & 12.90 & 67.1 & 70.56 \\
\hline 59 & 10.22 & 1.46 & 15.20 & 64.8 & 69.78 \\
\hline 60 & 18.65 & 2.53 & 19.84 & 60.16 & 61.35 \\
\hline
\end{tabular}


much greater than that of estimated yield and observed yield on study farms and indicated a scope to increase the yield of tomato. This analysis also indicated that higher the value of RAI gave higher estimated yield and smaller yield gap However, these results exclusively indicated to a production situation of tomato on the farmers' field where producers were not using the recommended doses of inputs and also management practices properly and completely in production system. Although the farmers could knew almost all identified inputs and management practices, yet they were not applying them properly and completely. As a result yield of tomato at the farmers' field was much lower than that of research station yield in the same growing season and made a sharp and big yield gap. This situation demands intensive extension work and also timely support with required inputs for minimizing the yield gap in production.

\section{Conclusion}

In the measure of "Real Adoption Impact (RAI)" of technologies on tomato production at farmers' field it was found that there was a positive and significant relationship between yield and the values of RAI. It implied that the higher value of RAI resulting from proper adoption of technologies in cultivation would give higher tomato yield. It was found that the expected yield gaps and observed yield gaps of the tomato farms were much greater than that of estimated yield and observed yield on study farms indicating a scope to increase the yield of tomato. The analysis also indicated that higher value of RAI gave higher estimated yield and smaller yield gap This situation demanded more effective extension work with tomato technologies so that the farmers could use the technologies completely and properly with their present capacity for improving tomato yield over the present yield level and to minimize the yield gaps. The capacity of the farmers to follow the recommended practices in tomato cultivation was identified from this study. As this study revealed that the farmers were using almost all recommended technologies of HRC in tomato cultivation, yet they were not adopting production technologies completely and properly in real operations indicating lower degree of proper adoption of technologies compared to research station. As a result the farmers could not reap the tomato yield at the expected level. However, the findings of the study help the planners to have a more precise and adequate future tomato production plan at national level. At the present level of farmers' capacity, any suggestions for better production would be more effective in tomato production to get a comparatively higher yield in per unit of area. This recommendation could also be made with the sensitivity analysis of RAI with respect to a particular technology while keeping all other technologies constant at the standard level.

The real adoption impact study will also be helpful to predict the yield of tomato at the farmers' level when one knows the capacity of the farmers to follow the recommended practices. Consequently any suggestion for 
improving the yield level can be made by the extension workers. However, the following specific recommendations may be made out of the findings of present study.

1. The tomato farmers in the study areas may increase the tomato yield through proper and complete use of technological inputs in production system.

2 As some new exotic/ improved tomato varieties those have been disseminated for production required intensive extension work at the farmers' level, the public authority at the same time must make attempts to make available the required inputs timely at farmers' needs. This effort would be helpful to stimulate the RAI to reduce the yield gap.

3. In greater spectrum, for commercialization of production and utilization of tomato with increasing demand at the market in Bangladesh context, some advertising activities are necessary for profitable cultivation of tomato crop for national interest.

\section{References}

Anon. (2004.) Summary crop Statistics, Bangladesh Bureau Statistics, Ministry of Planning, Govt. of the Peoples' Republic of Bangladesh, Dhaka. 1-4.

Anon. (2004) Krishi Projukti Hatboi (Hadbook on Agro-Technology), 3rd Ed. Bangladesh Agricultural Research Institute, Gazipur 1701. 1-559.
Barman, S. C and Mohd. (2005) Nazrul Islam. Real Adoption Impact Measure of Potato Technologies on Production at farmers' Level in Comilla District, Bangladesh. Indian Journal of Agricultural Economics., 60 (4) 677-685

Barman, S. C., and Shiblee, S.M. (2004-2005) An Analysis of Tomato Production and Its Prices and Consumption Level in Bangladesh. Annual Report, HRC, BARI, Joydebpur Gazipur 1701. 115-121.

Das. P.C. (1993) Vegetable Crops of India, 1st Ed. Kalyani Publishers, 1/1 Rajinder Nagar, Ludhiana 41008, India. 1- 219.

Hossain, M. (1981) Relationship of Selected Characteristics of the Jute Growers with their Adoption of Improved Practices of Jute Cultivation, M.Sc. (Ag. Ext. Ed.), Thesis, BAU, Mymensingh.

Razzaque M.A. (1977) Relationship of the selected characteristics of the farmers with the adoption of high yielding varieties (HYVs) of Rice in three Villages of Agricultural University Extension Project Area, M.Sc (Ag Ext. Ed.) thesis, BAU. Mymensingh.

Singh R. (1969) Fruits. National Book Trust, India. A.5. Green Park, New Delhi 110016. $1-221$.

Spiegel, M.R. (1961) Schaum's Outline Series, Theory and Problems of Statistics. Mc Grow Hill Book Company, New York, USA. 1359.

Received : August 28, 2006;

Accepted : March 27, 2007 